\title{
Native T1 is predictive of abnormal myocardial strain in hypertrophic cardiomyopathy
}

\author{
Patrick J Jensen ${ }^{2 *}$, Davis Vigneault ${ }^{1,3}$, Theodore Abraham ${ }^{4}$, Roselle Abraham ${ }^{4}$, David Bluemke ${ }^{1}$, Linda Chu ${ }^{5}$ \\ From 18th Annual SCMR Scientific Sessions \\ Nice, France. 4-7 February 2015
}

\begin{abstract}
Background
Hypertrophic cardiomyopathy (HCM) is an autosomal dominant inherited disease, characterized by myocardial wall thickening in the absence of other etiologies of hypertrophy. Histologically, HCM is characterized by myocyte hypertrophy and disarray with collagen deposition. Native T1 time of the myocardium is increased in the presence of myocardial fibrosis. The purpose of this study was to determine if greater $\mathrm{T} 1$ time was associated with abnormal myocardial wall strain.
\end{abstract}

\section{Methods}

Ninety-two patients were included in this cross-sectional study, all of whom had undergone CMR examination including steady state free precession (SSFP) cine and modified look-locker inversion recovery (MOLLI) imaging. Native T1 time was measured from short axis (SA) MOLLI images in the midslice of the left ventricle (LV). Strain, native T1 time, and wall thickness were measured at matched slice locations. Strain was measured using feature tracking of SSFP cine images (Multimodality Feature Tracking (MTT) algorithm v6.1.4826, Toshiba Medical Systems Corporation). Univariate comparisons were performed using Pearson's correlation and Student's t-test as appropriate, with $\mathrm{p}<0.05$ considered significant. In multivariate analysis, a generalized linear model was used to control for age, gender, LV mass index, maximum LV wall thickness, and LV ejection fraction. LV mass index was defined as LV mass divided by BSA. All statistical analyses were performed using R (v3.1.0, R Core Team) and RStudio (v0.97.551, RStudio).

\section{Results}

Of our cohort, $30 \%$ of the patients were women, $13 \%$ were African American, and 26\% were non-Caucasian.
The average age was 53 years. In multivariate analysis (Table), gender, ejection fraction, and native T1 time were predictive of myocardial strain ( $<<0.05$ for all), but myocardial wall thickness was not predictive of native T1 time. Greater native T1 time was associated with lower radial $(\mathrm{p}<0.01)$ and circumferential $(\mathrm{p}<0.01)$ strain magnitude. Per 10 milliseconds increase in native T1 time, the magnitudes of radial and mid circumferential strain decreased by $1.9 \%$ and $0.3 \%$, respectively.

\section{Conclusions}

In patients with hypertrophic cardiomyopathy, greater native $\mathrm{T} 1$ time was a more powerful predictor of decreased myocardial function than myocardial wall thickness. This suggests that the abnormality of myocardial strain in HCM may be related to the presence of diffuse myocardial fibrosis.

\section{Funding}

The investigators would like to acknowledge funding support from the NIH Intramural Research Program.

\section{Authors' details}

'Department of Radiology and Imaging Sciences, National Institutes of Health, Spring Lake, MI, USA. ${ }^{2}$ The College, University of Chicago, Chicago, MI, USA. ${ }^{3}$ Institute of Biomedical Engineering, University of Oxford, Oxford, UK. ${ }^{4}$ School of Medicine, Division of Cardiology, Johns Hopkins University, Baltimore, MD, USA. ${ }^{5}$ Department of Radiology and Radiological Science, Johns Hopkins University, Baltimore, MD, USA.

Published: 3 February 2015

doi:10.1186/1532-429X-17-S1-P263

Cite this article as: Jensen et al:: Native T1 is predictive of abnormal myocardial strain in hypertrophic cardiomyopathy. Journal of Cardiovascular Magnetic Resonance 2015 17(Suppl 1):P263.

${ }^{2}$ The College, University of Chicago, Chicago, MI, USA

Full list of author information is available at the end of the article 
Table 1 Multivariable analysis, relationship of Native T1 and covariates to myocardial strain by CMR

\begin{tabular}{|c|c|c|c|c|}
\hline \multirow[b]{2}{*}{ Covariate } & \multicolumn{2}{|c|}{ Radial Strain (\%) } & \multicolumn{2}{|c|}{ Mid Circumferential Strain (\%) } \\
\hline & B-value & P-value & B-value & P-value \\
\hline Native T1 (ms) & -0.19 & $<0.01$ & 0.03 & $<0.01$ \\
\hline LV Mass Index $(\mathrm{g} / \mathrm{m} \wedge 2)$ & 0.07 & 0.67 & 0.02 & 0.41 \\
\hline Gender (ref=male) & -10.9 & 0.075 & 2.2 & 0.021 \\
\hline Maximum LV Wall Thickness (mm) & -0.99 & 0.35 & 0.15 & 0.36 \\
\hline Age (yrs) & 0.01 & 0.93 & -0.02 & 0.38 \\
\hline Ejection Fraction (\%) & 1.31 & $<0.001$ & -0.25 & $<0.0001$ \\
\hline
\end{tabular}
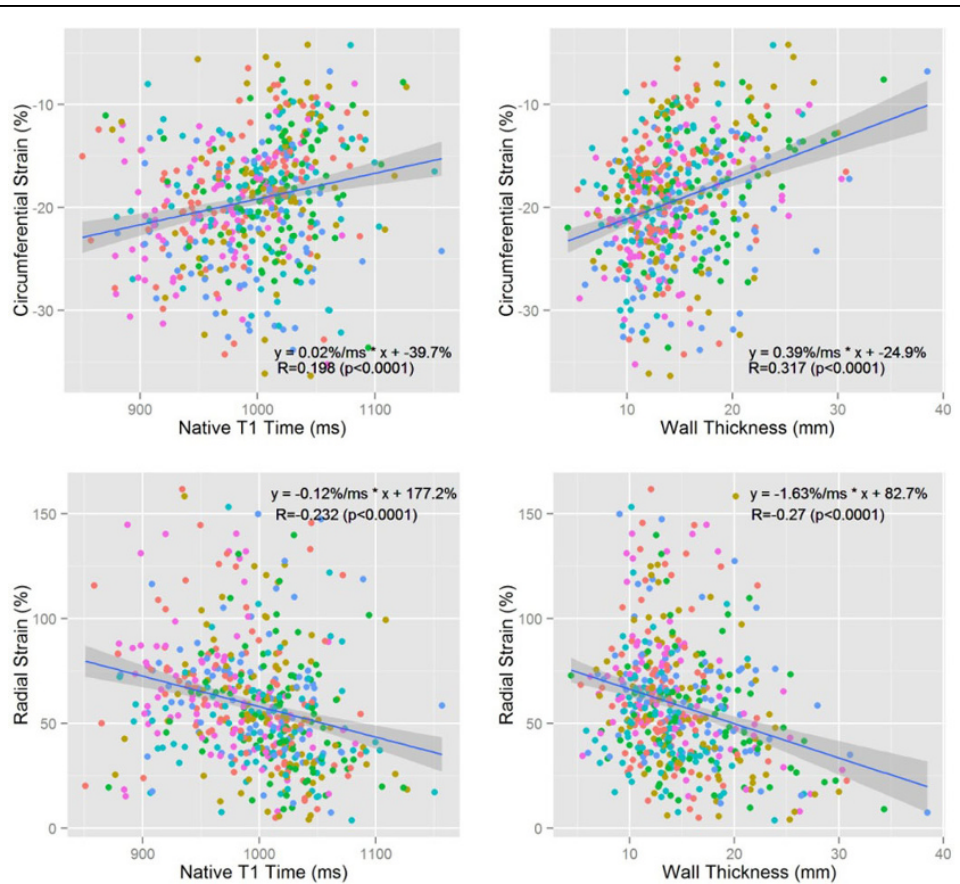

Segment

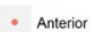

- Anteroseptal

- Inferoseptal

- Inferior

- Inferolateral

- Anterolateral

Figure 1 Segmental scatter plots comparing circumferential strain (top) and radial strain (bottom) to native T1 time (left) and wall thickness (right). Least squares regression nine and 95\% confidence interval are indicated. The equation of the regression line, as well as Pearson's $r$ coefficient, are indicated. 